\title{
Impact of different surgical protocols on dental development in oro-facial cleft children
}

\author{
Rosa Guagnano', Federica Romano², Ernesto Pepe ${ }^{3}$, Patrizia Defabianis ${ }^{1}$ \\ 'Department of Surgical Sciences C.I.R. Dental School - Section of Paediatric Dentistry, University of Turin, Turin 10126, Italy. \\ ${ }^{2}$ Department of Sciences C.I.R. Dental School - Section of Periodontology, University of Turin, Turin 10126, Italy. \\ ${ }^{3}$ Pediatric Plastic Surgery Division, City of Health and Science, Regina Margherita Children Hospital, Turin 10126, Italy.
}

Correspondence to: Prof. Patrizia Defabianis, Department of Surgical Sciences C.I.R. Dental School - Section of Paediatric Dentistry, University of Turin, Via Nizza 230, Turin 10126, Italy. E-mail: patrizia.defabianis@unito.it How to cite this article: Guagnano R, Romano F, Pepe E, Defabianis P. Impact of different surgical protocols on dental development
in oro-facial cleft children. Plast Aesthet Res 2020;7:37. http://dx.doi.org/10.20517/2347-9264.2020.21

Received: 13 Mar 2020 First Decision: 11 May 2020 Revised: 8 Jun 2020 Accepted: 25 Jun 2020 Published: 19 Jul 2020

Academic Editors: Carroll Ann Trotman, Xu Qian Copy Editor: Cai-Hong Wang Production Editor: Tian Zhang

\begin{abstract}
Aim: To determine the association between dental anomalies and type of facial cleft, gender, ethnicity and timing of hard palate repair surgery.

Methods: This observational study comprised a total of 85 non-syndromic cleft children (mean age $9.7 \pm 3.2$ years) of different ethnicity (68 Caucasians, 7 Asians, 4 Africans, 5 Hispanics and 1 Indian). Sixty-four patients were affected by lip palate cleft, 11 by lip alveolus cleft and 10 by palate cleft. Sixty-one children underwent delayed palate repair at 4.3 years of age, while 21 underwent early palate periosteoplasty at 7.2 months of age. Patients were examined clinically and radiologically to assess dental anomalies. Dental cavities were registered using $\mathrm{dmft/DMFT}$ indexes in primary and permanent dentition, while enamel defects were evaluated only in permanent teeth using Aine index.
\end{abstract}

Results: Tooth rotation and agenesis were the most common tooth anomalies affecting $59 \%$ and $42.2 \%$ of cleft patients, respectively. While a late closure of the cleft palate was associated with a higher number of rotations ( $P$ $=0.03$, an early surgical correction was associated to a higher frequency of tooth agenesis $(P=0.02)$, number of carious lesions in primary dentition $(P=0.002)$ and more severe enamel defects in permanent teeth $(P<0.01)$. A late palate repair increased 3.5 times the likelihood of having at least one rotated tooth $(P=0.034)$, while decreased the odds of having agenesis by $70 \%(P=0.029)$ compared to an early surgical repair.

Conclusion: Early surgical approaches seem to have more detrimental effects on dental development in both primary and permanent dentition than late surgical protocols. Dental abnormalities in cleft patients have complex etiology combining genetic and external factors and their prevalence can also depend on timing of hard palate surgery.

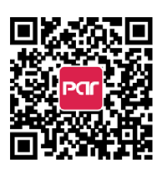


Keywords: Caries, cleft lip/palate, dental anomalies, hypoplasia, surgery

\section{INTRODUCTION}

Oro-facial clefts are a heterogeneous group of congenital malformations that recognise similar anomalies in development and fusion of facial processes during embryogenesis, which take place during the tenth week of pregnancy and involve soft and/or hard tissues of the skull.

Typical forms can be categorised into cleft lip with an incidence of 0.29 per 1000 births; cleft palate (CP), with an incidence of 0.31 per 1000 births; and cleft lip and palate (CLP) with an incidence of 0.48 per 1000 births ${ }^{[1]}$. Rare, atypical forms show an occurrence of 1 in over 100,000 births $^{[1]}$. In Italy, about 1 in 800 children are born with a facial cleft.

The aetiology is multifactorial and the causes of facial clefts are unknown but are thought to be caused by a combination of both genetic and environmental factors ${ }^{[2]}$. Lip palate cleft forms because of the maxillary and medial nasal processes fusion failure, disturbance in mesenchyme cells penetration between facial processes or vascular disruption ${ }^{[3]}$. In addition, the size of the facial processes - closely related to the ethnicity - can influence the facial morphology, increasing the susceptibility to develop a cleft: in Asian patients, for example, a smaller and flatter median nasal process, with a smaller third middle of the face and a more frequent trend to develop a skeletal third class, can result in a higher incidence of cleft $(2 / 100$ newborns) in comparison to Caucasians (1/1000 new-borns) and Afro-Americans (0.4/1000 new-borns) ${ }^{[4]}$.

Previous studies have reported a higher prevalence of dental abnormalities in the permanent dentition of cleft children than in the general population ${ }^{[5]}$. Tooth agenesis, supernumeraries and morphologic irregularities of the crown have been significantly associated with cleft size and severity ${ }^{[6]}$. Embriologically, the formation of tooth germs and the occurrence of cleft defects have a close relationship in terms of timing, thus factors leading to cleft could also affect the dental development ${ }^{[7,8]}$. In this context, recent studies have confirmed that genetic mutations (Interferon Regulatory Factor 6, Msh homeobox 1, Paired Box gene 9 and Transforming Growth Factor-beta) related to oral cleft lead to selective hypodontia and that Amelogenin $\mathrm{X}$ isoform gene, involved in the formation of the dental enamel, could also be involved in the development of clefts, suggesting a genetic association between dental anomalies and clefts ${ }^{[9,10]}$.

The literature regarding dental anomalies in cleft patients is heterogeneous, with dental anomalies rates varying more than two-fold. This discrepancy may be partially explained by patient selection criteria but may also suggest a role of external factors in enhancing the risk of tooth anomalies in cleft patients. While the impact of surgical cleft repair protocols on craniofacial growth has been widely investigated ${ }^{[11-13]}$, few studies have focused ${ }^{[14,15]}$ on their impact on tooth development. Primary cleft lip repair is usually performed at the age of 3-6 months. Palatal clefts closure is performed using two main techniques depending on surgical timing. The first technique is a two-stage protocol that consists of two separate operations: the first serves to close soft palate and is performed in the first/second year of age and the second serves to close the hard palate and is usually done before the pre-school age. The second technique, called periosteal plastic of the palate, is a one-stage palate repair, closing the soft and the hard palate at the same time before 12 months of age. Bone transplantation can be planned from infancy to adulthood to close the alveolar cleft.

We hypothesise that an early periosteal plastic surgery of the palate could affect dental development by reducing blood supply to developing tooth germs, thus causing more tooth agenesis and enamel defects compared to a delayed plastic surgery of the palate, performed later when the tooth germs are already formed. 
The aims of the present study were to assess the association between timing of hard palate surgery and dental anomalies in a sample of Italian cleft children and to investigate the relationship of dental abnormalities with gender, ethnicity and cleft type.

\section{METHODS}

All patients included in the present study were affected by non-syndromic facial cleft and were consecutively selected among outpatients referred for dental examination to the Section of Paediatric Dentistry, C.I.R. Dental School, University of Turin from April to July 2019. Patients were excluded if they were affected by lip or soft palate cleft, suffered from any disease associated with increased risk for dental anomalies or underwent previous extractions or fixed orthodontic treatment so that all dental surfaces could be accessible to the clinical examination and tooth agenesis as well as structural dental anomalies could not be considered iatrogenic.

The protocol of the present study was approved by the local Ethics Committee (No. 0038526), and written, informed consent was obtained from each patient or their parent or guardian. The investigation was performed according to the ethical principles of the Helsinki declaration.

Enrolled patients were classified into two groups depending on the timing the different surgical protocols were carried out. All patients who received one-stage periosteal plastic of the hard palate together with lip and soft palate repair were classified into the early periosteal palate plastic surgery group (EPP). This technique includes the treatment of maxillary defects and the closure of the lip at the age of 2-6 months. Cleft lip was repaired using a modified Tennison-Randall technique or a modified Mulliken technique. Cleft palate was repaired using Bardach technique or Von Langenbeck technique.

The patients included in the delayed palate repair surgery group (DPR) underwent first an infant orthopaedic treatment by the use of a Hotz neonatal plate followed at the age of 3-6 months by a lip repair procedure according to modified Millard or Noordhoff techniques. Soft palate repair was done at 8-10 months of age according to Widmaier-Perko technique, combined with V-Y repositioning of the soft palate without touching either the palatal artery or the palatal periosteum. Hard palate repair was performed at 4 years of age according to Schweckendiek technique with a mucoperiosteal flap. This is the current surgical treatment protocol to which cleft palate children treated at the Plastic Surgery Division of the Regina Margherita Children Hospital of Turin are submitted.

Data on age, gender, ethnicity, concomitant systemic pathologies, type and side of cleft and type and time of surgical corrections were collected from questionnaire and medical records. A specialist in paediatric dentistry evaluated the dental conditions of cleft children. Diagnosis of carious lesions was based on the criteria established by the World Health Organisation ${ }^{[16]}$. Each patient was given a score resulting from the sum of the decayed, missing and filled teeth either in primary (date index) or in permanent dentition (DMFT index). Patients with mixed dentition had two separate scores.

Disturbances of enamel mineralisation were examined on permanent teeth and recorded using the Aine rating scale where Grade I defines qualitative defects (opacities and discolorations), while Grades II, III and IV represent quantitative defects (hypoplasia) of increasing severity ${ }^{[17]}$.

Intraoral examination and panoramic radiographs were used to determine the following dental anomalies: number of impacted, missing, supernumerary or microdontic teeth, abnormalities in crown shape and ectopic eruption of permanent molars. Dental anomalies (fusion of deciduous teeth) were assessed only by intraoral examination for patients younger than 6 years of age. 


\section{Statistical analysis}

Data were recorded in a Microsoft Excel file and analysed using the Statistical Package for the Social Sciences (SPSS), version 24.0 (SPSS, Inc., Chicago, IL, USA).

Values of quantitative variables are presented as the mean \pm standard deviation, while values of categorical variables are presented as frequencies and percentages.

Data were first examined for normality by the Shapiro-Wilk test and if the data did not achieve normality, analyses were performed using non-parametric methods. The $\chi^{2}$ test was used to evaluate any potential association between categorical variables and the one-way analysis of variance or the Kruskal-Wallis test were used to assess differences of quantitative variables (Aine, Decayed Missing Filled Teeth index and decayed missing filled teeth index) between gender, ethnicity, cleft types and timings of palate surgery, as appropriate. When there were significant differences, pairwise multiple comparisons were carried out using the Scheffé test or the Dunn test. Logistic regression models were used to analyse the associations between surgical timings and dental anomalies. Estimates are shown as odds ratio (OR) and relative $95 \%$ confidence intervals (CIs) adjusted for gender, age, ethnicity and type of cleft.

All tests were two-tailed and p values less than 0.05 were considered statistically significant.

\section{RESULTS}

In total, 85 cleft subjects ( 51 male and 34 female) aged from 3 to 18 years (mean age $9.7 \pm 3.2$ years) were enrolled in the study, with 68 Caucasians (80\%), 7 Asians (8.2\%), 5 Hispanics (5.9\%), 4 Africans (4.7\%) and 1 Indian (1.2\%). All but 4 patients were systemically healthy, 1 suffered from heart disease, 1 referred a transient ischemic attack at birth, 1 referred hyposmia due to pituitary gland dysfunction and 1 was affected by a rare Tressier number 7 cleft and presented with bilateral cleft lip, right unilateral alveolar cleft and cleft of the upper maxillary molar region on the right side with macrostomia.

Sixty-four patients were affected by CLP: 29 on the left side (L-UCLP), 20 on the right side (R-UCLP) and 15 bilaterally (BCLP). Ten patients presented with CP and eleven with CLA, with 8 cases on the left side, 1 case on the right side and 1 case bilaterally. The left side was significantly more often affected than the right $(P<0.01)$. There was a statistical association between the type of cleft and gender with CLP more common in males $(67.2 \%)$ and $\mathrm{CP}$ in females $(70 \%)(P=0.047)$.

Sixty-one cleft patients (mean age $10.3 \pm 3.3$ years), belonging to the DPR group, had been treated at the Plastic Surgery Division of the Regina Margherita Children Hospital of Turin between January 2002 and December 2017. They underwent lip closure at a mean age of $6.1 \pm 2.3$ months, in combination with the use of a neonatal palatal plate, soft palate closure at a mean age of $12.7 \pm 4.3$ months, hard palate closure at $4.4 \pm 1.5$ years and bone grafting at a mean age of $12.0 \pm 1.6$ years.

Twenty-one patients (mean age $7.8 \pm 2.3$ years) in the EPP group were referred from other cleft centres in Italy: they all had been treated with a one-stage periosteal palatoplasty at a mean age of $7.2 \pm 6.5$ months between January 2006 and December 2015, without using the palatal plate. No information about cleft surgery was available for three patients.

Dental anomalies were assessed clinically and radiologically in 83 patients. The remaining two children were younger than 6 years of age and were submitted only to intraoral examination for assessing the presence of fused teeth. The frequencies of tooth anomalies by gender, ethnicity, cleft type and surgical protocol among subjects aged 6-18 years are summarised in Table 1, while the distribution by tooth type is described in Table 2. 
Table 1. Frequencies of tooth anomalies by gender, ethnicity, types of cleft and surgical protocols in cleft subjects aged 6-18 years

\begin{tabular}{|c|c|c|c|c|c|c|}
\hline Variables & $\begin{array}{c}\text { Rotations } \\
(n, \%)\end{array}$ & $\begin{array}{c}\text { Agenesis } \\
(n, \%)\end{array}$ & $\begin{array}{c}\text { Supernumerary } \\
(n, \%)\end{array}$ & $\begin{array}{c}\text { Shape anomalies } \\
(n, \%)\end{array}$ & $\begin{array}{c}\text { Impaction } \\
(n, \%)\end{array}$ & $\begin{array}{c}\text { Ectopic eruption } \\
(n, \%)\end{array}$ \\
\hline \multicolumn{7}{|l|}{ Gender } \\
\hline Male $(n=50)$ & $30(60 \%)$ & $20(40 \%)$ & $17(34 \%)$ & $8(16 \%)$ & $4(8 \%)$ & $2(4 \%)$ \\
\hline Female $(n=33)$ & $19(57.6 \%)$ & $15(45.5 \%)$ & $8(24.2 \%)$ & $9(27.3 \%)$ & $3(9.1 \%)$ & $4(12.1 \%)$ \\
\hline \multicolumn{7}{|l|}{ Ethnicity } \\
\hline Caucasian $(n=66)$ & $43(66.7 \%)^{\star}$ & $26(39.4 \%)$ & $18(27.3 \%)$ & $14(21.2 \%)$ & $5(7.6 \%)$ & $5(7.6 \%)$ \\
\hline Asian $(n=7)$ & $3(42.9 \%)$ & $5(71.4 \%)^{\star \star}$ & $2(28.6 \%)$ & $1(14.3 \%)$ & 0 & $1(14.3 \%)$ \\
\hline Hispanic $(n=5)$ & $2(40 \%)$ & $2(40 \%)$ & $2(40 \%)$ & $1(20 \%)$ & $1(20 \%)$ & 0 \\
\hline Indian $(n=1)$ & $1(100 \%)$ & 0 & 0 & 0 & 0 & 0 \\
\hline African $(n=4)$ & 0 & $2(50 \%)$ & $3(75 \%)^{\star}$ & $1(25 \%)$ & $1(25 \%)$ & 0 \\
\hline \multicolumn{7}{|l|}{ Cleft type } \\
\hline $\operatorname{CLP}(n=62)$ & $41(67.7 \%)$ & $28(45.2 \%)$ & $20(32.3 \%)$ & $13(12 \%)$ & $6(9.7 \%)$ & $5(8.1 \%)$ \\
\hline L-UCLP $(n=28)$ & $25(89.3 \%)^{\star \star}$ & $11(39.3 \%)$ & $6(21.4 \%)$ & $3(10.7 \%)$ & $4(14.3 \%)$ & $3(10.7 \%)$ \\
\hline R-UCLP $(n=20)$ & $10(50 \%)$ & $7(35 \%)$ & $5(25 \%)$ & $4(20 \%)$ & $1(5 \%)$ & $2(10 \%)$ \\
\hline $\mathrm{BCLP}(n=14)$ & $6(42.9 \%)$ & $10(71.4 \%)^{\star}$ & $9(64.3 \%)^{\star}$ & $6(42.9 \%)^{\star \star}$ & $1(7.1 \%)$ & 0 \\
\hline $\mathrm{CP}(n=10)$ & 0 & $3(30 \%)$ & 0 & 0 & 0 & $1(9.1 \%)$ \\
\hline $\operatorname{CLA}(n=11)$ & $8(72.7)^{\star}$ & $4(36.4 \%)$ & $5(45.5 \%)^{\star}$ & $4(36.4 \%)^{\star \star}$ & $1(9.1 \%)$ & \\
\hline \multicolumn{7}{|l|}{ Surgical protocol } \\
\hline $\operatorname{EPP}(n=20)$ & $8(40 \%)$ & $13(65 \%)^{\star}$ & $5(25 \%)$ & $3(15 \%)$ & $2(10 \%)$ & $1(5 \%)$ \\
\hline $\operatorname{DPR}(n=60)$ & $40(66.7 \%)^{\star}$ & $21(35 \%)$ & $20(33.3 \%)$ & $14(23.3 \%)$ & $4(6.7 \%)$ & $5(8.3 \%)$ \\
\hline
\end{tabular}

Values with superscript asterisks show statistically significant difference between groups: ${ }^{\star} P<0.05 ;{ }^{\star \star} P<0.01$. CLP: lip palate cleft; L-UCLP: left unilateral lip palate cleft; R-UCLP: right unilateral lip palate cleft; BCLP: bilateral lip palate cleft; CP: palate cleft; CLA: lip alveolar cleft; EPP: early palate periosteal plastic surgery; DPR: delayed palate repair surgery

Table 2. Frequencies of tooth anomalies and enamel defects by tooth type in cleft subjects aged 6-18 years

\begin{tabular}{|c|c|c|c|c|c|c|c|c|c|}
\hline \multirow[b]{2}{*}{ Tooth type } & \multirow[b]{2}{*}{$\begin{array}{l}\text { Rotation } \\
(n, \%)\end{array}$} & \multirow[b]{2}{*}{$\begin{array}{c}\text { Agenesis } \\
(n, \%)\end{array}$} & \multirow{2}{*}{$\begin{array}{c}\text { Supernumerary } \\
(n, \%)\end{array}$} & \multirow{2}{*}{$\begin{array}{c}\text { Shape } \\
\text { anomaly } \\
(n, \%)\end{array}$} & \multirow{2}{*}{$\begin{array}{c}\text { Impaction } \\
(n, \%)\end{array}$} & \multirow{2}{*}{$\begin{array}{c}\text { Ectopic } \\
\text { eruption } \\
(n, \%)\end{array}$} & \multicolumn{3}{|c|}{ Enamel Hypoplasia } \\
\hline & & & & & & & $\begin{array}{l}\text { Aine } 1 \\
(n, \%)\end{array}$ & $\begin{array}{l}\text { Aine } 2 \\
(n, \%)\end{array}$ & $\begin{array}{l}\text { Aine } 3 \\
(n, \%)\end{array}$ \\
\hline Upper Central incisors & $53(86.8 \%)$ & $4(6 \%)$ & $2(6.4 \%)$ & $2(9.5 \%)$ & $4(44.4 \%)$ & 0 & $14(50 \%)$ & $17(68 \%)$ & $4(80 \%)$ \\
\hline Upper Lateral Incisors & $8(13.1 \%)$ & $38(57.5 \%)$ & $24(77.4 \%)$ & $19(90.4 \%)$ & $1(11.1 \%)$ & 0 & $4(14.2 \%)$ & $3(12 \%)$ & $1(20 \%)$ \\
\hline Upper Canines & 0 & 0 & $1(3.2 \%)$ & 0 & $2(9.5 \%)$ & 0 & $2(7.1 \%)$ & 0 & 0 \\
\hline Upper premolars & 0 & $12(18.1 \%)$ & 0 & 0 & $1(11.1 \%)$ & 0 & 0 & $2(8 \%)$ & 0 \\
\hline Upper molars & 0 & 0 & $2(6.4 \%)$ & 0 & 0 & $7(100 \%)$ & $1(3.5 \%)$ & 0 & 0 \\
\hline Lower central incisors & 0 & 0 & $1(3.2 \%)$ & 0 & 0 & 0 & $3(10.7 \%)$ & 0 & 0 \\
\hline Lower lateral incisors & 0 & $4(6 \%)$ & $1(3.2 \%)$ & 0 & 0 & 0 & $1(3.5 \%)$ & 0 & 0 \\
\hline Lower canines & 0 & 0 & 0 & 0 & 0 & 0 & 0 & 0 & 0 \\
\hline Lower premolars & 0 & 6 & 0 & 0 & 0 & 0 & 0 & $1(4 \%)$ & 0 \\
\hline Lower molars & 0 & $2(3 \%)$ & $1(3.2 \%)$ & 0 & $1(11.1 \%)$ & 0 & $3(10.7 \%)$ & $2(8 \%)$ & 0 \\
\hline Total & 61 & 66 & 31 & 21 & 9 & 7 & 28 & 25 & 5 \\
\hline
\end{tabular}

No significant gender difference in the prevalence of tooth anomalies was observed, while ethnicity, cleft type and surgical timing were statistically significantly related to their frequency. Rotation was the most common development anomaly of dentition (59\%), affecting one tooth in 37 patients (44.6\%) and two teeth in 12 patients $(14.5 \%)$. Caucasians and DPR patients exhibited tooth rotations more often than other racial groups as well as more often than EPP patients (both $P=0.03$ ). Rotations were also more frequent in L-UCLP and CLA, while they were absent in CP patients $(P<0.01)$. The upper central left permanent incisor was the most frequently affected tooth $(50.8 \%)$.

Agenesis affected one tooth in 19 subjects (22.9\%), two/three teeth in $14(16.9 \%)$ subjects and four/five teeth in 2 subjects $(2.4 \%)$. It was more frequent in Asians $(P<0.01)$ and BCLP subjects $(P=0.014)$ and those submitted to EPP $(P=0.02)$. Upper lateral incisors were the teeth more commonly involved in this anomaly.

One or two supernumerary teeth were found in 25 patients (30.1\%), involving more often the upper lateral incisors. Their frequency was higher in Africans $(P=0.04)$, CLA $(45.5 \%)$ and CLP $(32.3 \%)$ subjects, in particular those with R-UCLP $(P=0.01)$. 
Table 3. Enamel defects on permanent teeth (Aine index) by gender, ethnicity types of cleft, and surgical protocols (mean \pm SD)

\begin{tabular}{|c|c|c|c|c|}
\hline \multirow{2}{*}{ Variables } & \multicolumn{4}{|c|}{ Aine } \\
\hline & 0 & 1 & 2 & 3 \\
\hline \multicolumn{5}{|l|}{ Gender } \\
\hline Male $(n=42)$ & $14.0 \pm 7.0$ & $0.4 \pm 1.1$ & $0.1 \pm 0.4$ & $0.1 \pm 0.2$ \\
\hline Female $(n=23)$ & $13.3 \pm 8.5$ & $1.1 \pm 2.8$ & $0.5 \pm 0.9$ & $0.1 \pm 0.4$ \\
\hline \multicolumn{5}{|l|}{ Ethnicity } \\
\hline Caucasian $(n=53)$ & $14.7 \pm 7.8^{\star}$ & $0.7 \pm 1.7$ & $0.3 \pm 0.6$ & $0.1 \pm 0.2$ \\
\hline Asian $(n=5)$ & $4.2 \pm 1.8$ & $0.4 \pm 0.9$ & $0.2 \pm 0.5$ & $0.0 \pm 0.0$ \\
\hline Hispanic $(n=3)$ & $13.0 \pm 7.8$ & $0.0 \pm 0.0$ & $0.3 \pm 0.6$ & $1.0 \pm 0.9$ \\
\hline Indian $(n=1)$ & 10.0 & 0.0 & 0.0 & 0.0 \\
\hline African $(n=3)$ & $10.3 \pm 10.1$ & $5.0 \pm 8.7$ & $2.3 \pm 2.1$ & $0.0 \pm 0.0$ \\
\hline \multicolumn{5}{|l|}{ Cleft type } \\
\hline $\operatorname{CLP}(n=50)$ & $14.0 \pm 8.3$ & $1.0 \pm 0.7^{*}$ & $0.4 \pm 0.8$ & $0.1 \pm 0.4$ \\
\hline $\mathrm{CP}(n=5)$ & $9.6 \pm 1.8$ & $0.0 \pm 0.0$ & $0.0 \pm 0.0$ & $0.0 \pm 0.0$ \\
\hline $\operatorname{CLA}(n=10)$ & $13.3 \pm 8.2$ & $0.2 \pm 0.4$ & $0.4 \pm 0.7$ & $0.1 \pm 0.3$ \\
\hline \multicolumn{5}{|l|}{ Surgical protocol } \\
\hline $\operatorname{EPP}(n=16)$ & $15.3 \pm 7.9^{\star \star}$ & $1.0 \pm 2.7$ & $0.5 \pm 0.9^{\star \star}$ & $0.0 \pm 0.2$ \\
\hline $\operatorname{DPR}(n=49)$ & $8.1 \pm 5.8$ & $0.2 \pm 0.6$ & $0.1 \pm 0.4$ & $0.2 \pm 0.6$ \\
\hline
\end{tabular}

Values with superscript asterisks show statistically significant difference between groups: ${ }^{\star} P<0.05$; CLP: lip palate cleft; CP: palate cleft; CLA: lip alveolar cleft; EPP: early palate periosteal plastic; DPR: delayed palate plastic

Tooth impaction was observed in seven subjects (8.4\%), involving one tooth in five subjects (6\%) and two teeth in two subjects $(2.4 \%)$. The upper central incisors $(3.7 \%)$ and canines $(1.8 \%)$ were the most frequently impacted teeth. A minority of subjects (7.2\%) presented with ectopic eruption of permanent molars, with two teeth erupted ectopically in only one case.

Morphological abnormalities of dental crowns were detected in 17 subjects (20.5\%) affecting one (15.6\%) or two teeth $(4.8 \%)$ and were observed more frequently in B-CLP subjects $(42.9 \%)(P=0.001)$. Twelve patients (14.5\%) exhibited microdontic upper lateral incisors and five patients $(6.0 \%)$ the fusion of two deciduous teeth (lower lateral incisor with canine $4.7 \%$ and upper central incisors together $1.2 \%$ ). No primary fused teeth were found in children younger than 6 years of age.

Logistic analysis adjusted for gender, age, ethnicity and cleft type showed a significant association between timing of palate surgery, tooth rotation and agenesis. DPR children exhibited a 3.5-fold higher likelihood of having at least one rotated tooth than EPP children ( $\mathrm{OR}=3.50$, 95\%CI: 1.10-11.13, $P=0.034)$ but lower likelihood of having at least one agenesis tooth $(\mathrm{OR}=0.26,95 \% \mathrm{CI}: 0.08-0.87, P=0.029)$.

As summarised in Table 3, in total 984 permanent teeth were also examined in 65 patients for enamel defects: 13 patients (20\%) showed opacities and discolorations (Aine 1), $15(23 \%)$ mild (Aine 2) and $4(6 \%)$ evident structural defects (Aine 3). No Aine 4 defects were found. As described in Table 3, CLP subjects showed higher mean number of enamel defects (Aine 1) compared to CLA and CP subjects $(P=0.012)$. More severe enamel defects (Aine 3 ) were also observed in patients submitted to EPP compared to those who underwent DPR $(P<0.01)$. The upper central and lateral incisors were the most frequently involved teeth [Table 1].

Table 4 summarises data on caries experience in both primary and permanent dentition. The mean $\mathrm{dmft}$ index was higher in EPP subjects compared to DPR subjects $(P=0.002)$, while no statistically significant differences were observed among males and females, ethnic groups and cleft types. In addition, no statistically significant differences were observed for DMTF index scores.

\section{DISCUSSION}

A recent systematic review on frequency of dental anomalies in cleft patients emphasised that data in the literature are difficult to compare because of the heterogeneity in terms of surgical cleft closure techniques, 
Table 4. Dental caries in primary (dmft) and permanent teeth (DMFT) by gender, ethnicity types of cleft, and surgical protocols in the study sample (mean \pm SD)

\begin{tabular}{lll}
\hline Variables & dmft & DMFT \\
\hline Gender & & \\
Male $(n=51)$ & $2.4 \pm 2.9$ & $0.9 \pm 1.5$ \\
Female $(n=34)$ & $1.8 \pm 2.8$ & $1.1 \pm 2.1$ \\
Ethnicity & & \\
Caucasian $(n=68)$ & $1.9 \pm 2.9$ & $1.8 \pm 0.4$ \\
Asian $(n=7)$ & $3.5 \pm 2.6$ & $0.4 \pm 0.5$ \\
Hispanic $(n=5)$ & $4.0 \pm 2.9$ & $1.0 \pm 1.1$ \\
Indian $(n=1)$ & 4.0 & 0.0 \\
African $(n=4)$ & $1 \pm 1.1$ & $1 \pm 1.1$ \\
Cleft type & & $1.1 \pm 2.4$ \\
CLP $(n=64)$ & $2.2 \pm 1.0$ & $0.3 \pm 0.9$ \\
CP $(n=10)$ & $2.5 \pm 1.0$ & $0.7 \pm 1.0$ \\
CLA $(n=11)$ & $1.5 \pm 0.9$ & $0.8 \pm 1.2$ \\
Surgical protocol & & $1.1 \pm 1.9$ \\
EPP $(n=21)$ & $3.9 \pm 3.0^{*}$ & $1.5 \pm 2.6$ \\
DPP $(n=61)$ & & \\
\hline
\end{tabular}

Values with superscript asterisks show statistically significant difference between groups: ${ }^{\star} P<0.05$. CLP: lip palate cleft; CP: palate cleft; CLA: lip alveolar cleft; EPP: early palate periosteal plastic; DPR: delayed palate plastic

interval time between surgeries, study design and length of follow-up ${ }^{[6]}$. Moreover, little information is available on the potential effects that different surgical timings of hard palate repair, early or late, could have on dental development ${ }^{[14,15]}$. It is indeed well known that all types of surgical repair of oro-facial clefts are detrimental to maxillary growth and development of permanent teeth.

The present results suggest that surgical timing could impact only on the frequency of rotations and agenesis, while other tooth anomalies were not significantly affected. Interestingly, a late surgical repair increased 3.5 times the likelihood of having at least one rotated tooth, while decreased the odds of having agenesis by $70 \%$.

A delayed surgical repair of the cleft palate could favour tooth rotation because of the persistence of lack of bone and space for incisor eruption ${ }^{[18]}$. This situation could even be worsened by the concomitant presence of supernumeraries in the cleft area. It should be considered that tooth rotation is the most frequently observed anomaly at the cleft side, mainly in subjects with L-UCLP, especially affecting the maxillary central incisors.

By contrast, tooth agenesis was found more commonly in children who underwent early cleft palate surgical closure. Surgical trauma during early palatal periosteal plastic and reduction of the blood supply due to tissue tension and excessive scarring associated with palatal defects, as well as absence of early orthopaedic treatment to optimise the position of maxillary fragments, have been suggested as external causes of agenesis and enamel defects in upper permanent incisors ${ }^{[14]}$.

Notably, we observed a prevalence of tooth agenesis of $42.2 \%$, that was lower than that reported in the literature, ranging from $45 \%$ to $67.6 \%{ }^{[19-23]}$. This lower frequency could be explained by the fact that most of the patients were Caucasian, an ethnicity with a low incidence of agenesis, and underwent a late surgical closure of the hard palate, at 4 years of age or older. Tooth agenesis affected more frequently BCLP subjects (71.4\%), in which the alveolar defect is more severe than in other cleft types. According to the literature, upper lateral incisors were the more common missing teeth $(40 \%)$, followed by upper and lower second premolars (18\%), lower left incisors and lower second molars $(2 \%)^{[6]}$.

Morphological abnormalities of dental crowns were observed in $20.5 \%$ of the patients, being the lateral incisors the most frequently affected teeth. In particular, microdontia was found in $14.5 \%$ of cleft children, 
a lower percentage compared to that reported in the literature, ranging from $18 \%$ to $37 \%^{[19-24]}$. Conversely, only a minority of patients (6\%) showed primary fused teeth, as previously observed by Suzuki et al. ${ }^{[21]}$. Notably, fusion of deciduous teeth in the present study was always followed by the agenesis of the permanent teeth.

Tooth impaction was observed in $8.4 \%$ of the patients and ectopic eruption of molars in $7.2 \%$ of the cases. These percentages were lower compared to data reported in the literature, which varied from $10 \%$ to $50 \%{ }^{[19-25]}$ and from $15 \%$ to $28 \%{ }^{[26]}$, respectively.

Worth et al. ${ }^{[27]}$ reported that the dental caries prevalence in cleft lip palate patients is higher than that observed in healthy children, in both deciduous and permanent dentition, with a pooled mean difference in dmft of 0.63 (95\%CI: 0.47-0.79) and in DMFT of 0.28 (95\%CI: 0.22-0.34). In the present cleft population, caries experience was two-fold higher in primary than in permanent teeth. Dental anomalies in the deciduous dentition may predispose the affected teeth to greater accumulation of bacterial plaque and consequently to dental caries ${ }^{[28]}$. Thus, counselling and follow-up are important to maintain the integrity of teeth in order to maintain the supportive bone structures that may be defective at the cleft area.

Finally, in line with previous studies ${ }^{[29]}$, enamel defects, varying from opacity and discoloration to mild and evident structural changes, were more frequently observed in upper left lateral and central permanent incisors. In agreement with Korolenkova et al. ${ }^{[14]}$, we observed a statistically significant association between early periosteal plastic surgery of the palate and higher number of carious lesions in deciduous teeth or severe degree of enamel defects in permanent dentition and a higher prevalence of permanent tooth agenesis. A possible explanation could be that an early surgery on hard tissues, when primary teeth are erupting and permanent teeth (particularly the incisors) are developing, can interfere with blood supply of dental buds, resulting in more enamel defects up to agenesis. Indeed, the lower percentage of damage to dental enamel reported in our study $\left(49 \% v\right.$ s. $87.9 \%{ }^{[29]}$ ) could be related to the fact that most of the patients underwent late surgical repair of the hard palate, with less impact on dental development, while the most severe consequences were observed in patients treated early in life.

The limitation of the present study is the heterogeneity of enrolled patients in terms of type of cleft, ethnicity and surgical procedures for cleft repair. In addition, the study sample was a convenience sample, but it provided significant results about the association between surgical timings and dental anomalies in cleft lip palate patients, suggesting that prevalence of dental abnormalities may also depend on treatment protocol.

Further multi-centre studies with larger numbers of cleft children should be performed to investigate timing, type of surgical cleft repairs, clinical and patient-related outcomes in order to identify the most appropriate surgical approach to optimise both speech outcomes and maxillary bone development while at the same time limiting the detrimental impact on both primary and permanent dentition. While early hard palate repair improves speech production, delayed repair allows for better maxillary growth ${ }^{[30]}$. Intensive speech therapy directed at the correction of articulation errors should be implemented as soon as possible to improve overall communication.

Interdisciplinary management and proper follow-up of these patients are crucial and paediatric dentists must be conscious about the dental needs of these subjects in order to improve their quality of life.

\section{DECLARATIONS}

\section{Authors' contributions}

Made substantial contributions to conception and design of the study: Guagnano R, Defabianis P Performed data acquisition and provided administrative, technical, and material support: Guagnano R 
Performed data analysis and interpretation: Romano F

Performed cleft surgeries: Pepe E

Made substantial contributions in writing the paper: Guagnano R, Defabianis $\mathrm{P}$

\section{Availability of data and materials}

Not applicable.

\section{Financial support and sponsorship}

None.

\section{Conflicts of interest}

All authors declared that there are no conflicts of interest.

\section{Ethical approval and consent to participate}

The Institutional Ethics Committee of the "AOU Città della Salute e della Scienza", of Turin (Italy) approved the research protocol (No. 0038526). The study was performed according to the ethical principles of the Helsinki declaration. Written and informed consent was obtained from participants or their parents or legal guardians.

\section{Consent for publication}

Not applicable.

\section{Copyright}

(c) The Author(s) 2020.

\section{REFERENCES}

1. Tolarova MM, Cervenka J. Classification and birth prevalence of orofacial cleft. Am J Med Genet 1998;75:126-37.

2. Raut JR, Simeone RM, Tinker SC, Canfield MA, Day RS, et al. Proportion of orofacial clefts attributable to recognized risk factors. Cleft Palate Craniofac J 2019;56:151-8.

3. Rice DP. Craniofacial anomalies: from development to molecular pathogenesis. Curr Mol Med 2005;5:699-722.

4. Gundlach KK, Maus C. Epidemiological studies on the frequency of clefts in Europe and world-wide. J Craniomaxillofac Surg 2006;34:1-2.

5. da Silva AP, Costa B, de Carvalho Carrara CF. Dental anomalies of number in the permanent dentition of patients with bilateral cleft lip: radiographic study. Cleft Palate Craniofac J 2008;45:473-6.

6. Tannure PN, Oliveira CA, Maia LC, Vieira AR, Granjeiro JM, et al. Prevalence of dental anomalies in nonsyndromic individuals with cleft lip and palate: a systematic review and meta-analysis. Cleft Palate Craniofac J 2012;49:194-200.

7. Stahl F, Grabowski R, Wigger K. Epidemiology of Hoffmeister's "genetically determined predisposition to disturbed development of the dentition" in patients with cleft lip and palate. Cleft Palate Craniofac J 2006;43:457-65.

8. Howe BJ, Cooper ME, Vieira AR, Weinberg SM, Resick JM, et al. Spectrum of dental phenotypes in nonsyndromic orofacial clefting. J Dent Res 2015;94:905-12.

9. Simioni M, Araujo TK, Monlleo IL, Maurer-Morelli CV, Gil-da-Silva-Lopes VL. Investigation of genetic factors underlying typical orofacial clefts: mutational screening and copy number variation. J Hum Genet 2015;60:17-25.

10. Oliveira FV, Dionísio TJ, Neves LT, Machado MA, Santos CF, et al. Amelogenin gene influence on enamel defects of cleft lip and palate patients. Braz Oral Res 2014;28:1-6.

11. Stoltz JF, Nicolas A. Study of amino groups of the human platelet membrane. Acta Haematol 1978;60:304-9.

12. Salgado KR, Wendt AR, Fernandes Fagundes NC, Maia LC, Normando D, et al. Early or delayed palatoplasty in complete unilateral cleft lip and palate patients? A systematic review of the effects on maxillary growth. J Craniomaxillofac Surg 2019;47:1690-8.

13. Farronato G, Kairyte L, Giannini L, Galbiati G, Maspero C. How various surgical protocols of the unilateral cleft lip and palate influence the facial growth and possible orthodontic problems? Which is the best timing of lip, palate and alveolus repair? Literature review. Stomatologija 2014;16:53-60.

14. Korolenkova MV, Starikova NV, Udalova NV. The role of external aetiological factors in dental anomalies in non-syndromic cleft lip and palate patients. Eur Arch Paediatr Dent 2019;20:105-11.

15. Korolenkova MV, Starikova NV, Ageeva LV. Risk factors for teeth aplasia and hypoplasia in cleft lip and palate children. Stomatologiia (Mosk) 2016;95:59-62. 
16. World Health Organization. (1997). Oral health surveys : basic methods, 4th ed. World Health Organization. Available from: https://apps. who.int/iris/handle/10665/41905 [Last accessed on 16 Jul 2020]

17. Aine L, Mäki M, Collin P, Keyriläinen O. Dental enamel defects in celiac disease. J Oral Pathol Med 1990;19:241-5.

18. Smahel Z, Tomanová M, Müllerová Z. Position of upper permanent central incisors prior to eruption in unilateral cleft lip and palate. Cleft Palate Craniofac J 1996;33:219-24.

19. Tortora C, Meazzini MC, Garattini G, Brusati R. Prevalence of abnormalities in dental structure, position, and eruption pattern in a population of unilateral and bilateral cleft lip and palate patients. Cleft Palate Craniofac J 2008;45:154-62.

20. Germec Cakan D, Nur Yilmaz RB, Bulut FN, Aksoy A. Dental anomalies in different types of cleft lip and palate: is there any relation? J Craniofac Surg 2018;29:1316-21.

21. Suzuki A, Takahama Y. The maxillary lateral incisor of subject with cleftlip and/or palate (part 1). Cleft Palate Craniofac J 1992;29:376-9.

22. Aizenbud D, Camasuvi S, Peled M, Brin I. Congenitally missing teeth in the Israeli cleft population. Cleft Palate Craniofac J 2005;42:314-7.

23. Al Jamal GA, Hazza'a AM, Rawashdeh MA. Prevalence of dental anomalies in a population of cleft lip and palate patients. Cleft Palate Craniofac J 2010;47:413-20.

24. Suzuki A, Nakano M, Yoshizaki K, Yasunaga A, Haruyama N, et al. A longitudinal study of the presence of dental anomalies in the primary and permanent dentitions of cleft lip and/or palate patients. Cleft Palate Craniofac J 2017;54:309-20.

25. Hinrichs JE, El-deeb ME, Waite DE, Bevis RR, Bandt CL. Periodontal evaluation of canines erupted through grafted alveolar cleft defects. J Oral Maxillofac Surg 1984;42:717-21.

26. da Silva Filho OG, De Albuquerque MV, Kurol J. Ectopic eruption of maxillary first permanent molars in children with cleft lip. Angle Orthod 1996;66:373-80.

27. Worth V, Perry R, Ireland T, Wills AK, Sandy J, et al. Are people with an orofacial cleft at a higher risk of dental caries? A systematic review and meta-analysis. Br Dent J 2017;223:37-47.

28. Cheng LL, Moor SL, Ho CT. Predisposing factors to dental caries in children with cleft lip and palate: a review and strategies for early prevention. Cleft Palate Craniofac J 2007;44:67-72.

29. Shen CA, Guo R, Li W. Enamel defects in permanent teeth of patients with cleft lip and palate: a cross-sectional study. J Int Med Res 2019;47:2084-96

30. Shaffer AD, Ford MD, Losee JE, Goldstein J, Costello BJ, et al. The association between age at palatoplasty and speech and language outcomes in children with cleft palate: an observational chart review study. Cleft Palate Craniofac J 2020;57:148-60. 